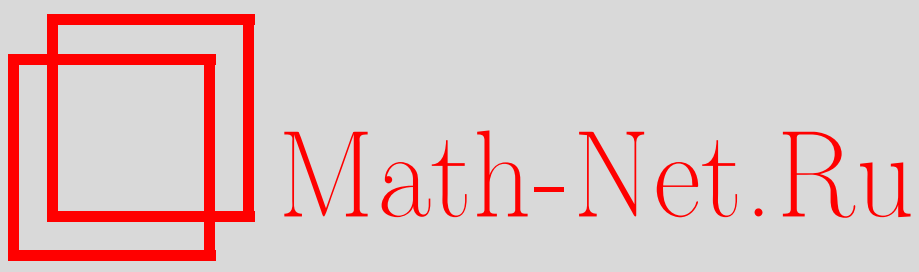

А. Л. Гладков, О поведении решений некоторых квазилинейных параболических уравнений со степенными нелинейностями, Матем. сб., 2000, том 191, номер 3, 2542

DOI: https://doi.org/10.4213/sm462

Использование Общероссийского математического портала Math-Net.Ru подразумевает, что вы прочитали и согласны с пользовательским соглашением

http://www.mathnet.ru/rus/agreement

Параметры загрузки:

IP: 54.237 .206 .68

26 апреля 2023 г., $02: 41: 22$ 


\section{А. Л. Гладков \\ О поведении решений некоторых квазилинейных параболических уравнений со степенными нелинейностями}

В работе рассматривается задача Коши с неотрицательной непрерывной начальной функцией для уравнения

$$
u_{t}=\Delta u^{m}-u^{p}, \quad(x, t) \in S=\mathbb{R}^{N} \times \mathbb{R}_{+},
$$

где $0<p<1, p<m$. Для обобщенных решений этой задачи с растущими на бесконечности начальными данными получен ряд теорем об их поведении при $t \rightarrow \infty$.

Библиограффия: 11 названий.

\section{§1. Введение}

В статье рассматриваются неотрицательные решения задачи Коши для уравнения

$$
u_{t}=\Delta u^{m}-u^{p}, \quad(x, t) \in S=\mathbb{R}^{N} \times \mathbb{R}_{+},
$$

с начальным условием

$$
u(x, 0)=u_{0}(x), \quad x \in \mathbb{R}^{N},
$$

где $0<p<1, p<m, \mathbb{R}_{+}=(0,+\infty), u_{0}(x)$ - неотрицательная непрерьвная функция, которая может расти на бесконечности.

Уравнение (1.1) возникает как модельное во многих областях физики (см., например, [1] и имеющуюся там библиографию). В частности, оно описывает фильтрацию жидкости через однородную пористую среду, диффузию газа и распространение тепла в нелинейной среде. Присутствие младшего члена в уравнении указывает на наличие в среде поглощения. Как хорошо известно, задача (1.1), (1.2) может не иметь классического решения. Поэтому введем понятие обобщенного решения.

ОПРЕДЕЛЕниЕ 1 . Неотрицательная непрерывная в $S$ функция $u(x, t)$ называется обобщенным решением уравнения (1.1) в $S$, если $u(x, t)$ удовлетворяет интегральному тождеству

$$
\int_{t_{1}}^{t_{2}} \int_{\mathbb{R}^{N}}\left\{u f_{t}+u^{m} \Delta f-u^{p} f\right\} d x d t-\left.\int_{\mathbb{R}^{N}} u f\right|_{t=t_{1}} ^{t=t_{2}} d x=0
$$

для всех $t_{i}$ таких, что $0<t_{1}<t_{2}$, и любой неотрицательной функции $f(x, t) \in$ $C_{x, t}^{2,1}(S)$, имеюшей компактньй носитель в $S$.

При замене в $(1.3)$ знака $=$ на знак $\geqslant(\leqslant)$ получаем определение обобщенного субрешения (суперрешения) уравнения (1.1) в $S$. Аналогично вводятся эти понятия в слое $S_{T}=\mathbb{R}^{N} \times(0, T)$. 
ОПРЕДЕЛЕНИЕ 2. Функцию $u(x, t)$ назовем обобщенным решением задачи Ко$u и(1.1),(1.2)$ в $S\left(S_{T}\right)$, если она является обобшенным решением уравнения (1.1) в $S\left(S_{T}\right)$, непрерьвна в $\bar{S}\left(\bar{S}_{T} \backslash \mathbb{R}^{N} \times\{T\}\right)$ и выполнено условие (1.2).

В случае $m=1$ результаты, аналогичные представленным в статье, получены в [2]. В настолщей работе анализируется эволюция решений задачи (1.1), (1.2). При этом используются многие идеи и методы работы [2]. Для удобства условимся в дальнейшем обозначать положительные и неотрицательные постоянные через $M_{i}$ и $\alpha_{i}$ соответственно. Обозначим через $K\left(S_{T}\right)$ класс неотрицательных функций $v(x, t)$, для которых в $S_{T}$ выполнено неравенство:

$$
v(x, t) \leqslant M_{1}\left(\alpha_{1}+|x|\right)^{2 /(m-1)} .
$$

Здесь постоянные $M_{1}$ и $\alpha_{1}$ могут зависеть от функции $v(x, t)$.

ТеОРема 1. Пусть $m>1 u u_{0}(x)$ при некоторых постоянных $M_{2} u \alpha_{2}$ удовлетворяет неравенству

$$
u_{0}(x) \leqslant M_{2}\left(\alpha_{2}+|x|\right)^{(2-\epsilon) /(m-1)} .
$$

Если $\epsilon>0$ в (1.4), то для любого значения $T>0$ задача (1.1), (1.2) имеет обобщенное решение в $S_{T} u(x, t) \in K\left(S_{T}\right)$. B случае $\epsilon=0$ при достаточно малом значении $T>0$ задача (1.1), (1.2) разрешима в слое $S_{T}$ u $u(x, t) \in$ $K\left(S_{T}\right)$. Обобщенное решение единственно в произвольном слое $S_{T}$ в классе функиий $K\left(S_{T}\right)$.

ТЕОРема 2. Пусть $0<m<1$. Тогда для любой неотрицательной начальной функиии $u_{0}(x)$ задача $(1.1),(1.2)$ имеет единственное обобщенное решение в $S$.

Для одномерного случая теорема 1 доказана в [3]. В многомерном случае доказательство аналогичное. Теорема 2 доказывается так же, как, например, в [4]-[6]. Сушествование и единственность решения задачи (1.1), (1.2) с $m>1$ и $p>1$ установлены в [6]-[9].

Если специально не будет оговорено, то через $o(z(x))$ обозначаются в дальнейшем бесконечно малые при $|x| \rightarrow+\infty$ функции более высокого порядка, чем $z(x)$. Определим время “остывания" $T_{E}(x)$ обобщенного решения в точке $x \in \mathbb{R}^{N}$ следуюшим образом:

$$
T_{E}(x)=\sup \{t>0: u(x, t)>0\} .
$$

Очевидно, что $T_{E}(x)$ может равняться бесконечности, но сушествуют случаи, когда время "остывания" конечно для всех $x \in \mathbb{R}^{N}$. Это происходит, например, если диффузия отсутствует в уравнении или достаточна мала по сравнению с поглощением. Баланс между диффузией и поглощением наблюдается в следующей ситуации:

$$
\Delta u^{m}=u^{p} .
$$

Как легко можно заметить, для любого значения $a \in \mathbb{R}^{N}$ уравнение (1.5) имеет точное решение

$$
u(x, a)=c_{N}|x-a|^{2 /(m-p)}, \quad c_{N}=\left\{\frac{(m-p)^{2}}{2 m[m N+p(2-N)]}\right\}^{1 /(m-p)} .
$$

Постоянная $c_{N}$ в (1.6) является критической для “остывания" обобщенного решения во всех точках пространства $\mathbb{R}^{N}$. Более точно, справедлива 
ТЕОРемА 3. 1) Предположим, что $u_{0}(x)$ удовлетворяет неравенству

$$
u_{0}(x) \leqslant A(|x|)+B|x-a|^{2 /(m-p)}
$$

для некоторого $а \in \mathbb{R}^{N}$, где $A(|x|)=o\left(|x|^{2 /(m-p)}\right)$ u

$$
0 \leqslant B<c_{N}
$$

Тогда для любого $y \in \mathbb{R}^{N}$

$$
T_{E}(y) \leqslant \frac{1}{1-p} \inf _{0<\varepsilon<c_{N}-B} \frac{[M(R(\varepsilon))]^{1-p}}{\left[1-\left((B+\varepsilon) / c_{N}\right)^{m}\right]^{\frac{m-p}{m}}}
$$

$n p u m>1 u$

$$
T_{E}(y) \leqslant \frac{1}{1-p} \inf _{0<\varepsilon<c_{N}-B}\left\{\frac{M(R(\varepsilon))}{1-\left((B+\varepsilon) / c_{N}\right)^{\frac{m-p}{1-p}}}\right\}^{1-p}
$$

при $p<m<1$. Здесь $M(R)=\max _{|x-a| \leqslant R} u_{0}(x)$, a $R(\varepsilon)-$ максимальный корень уравнения

$$
(B+\varepsilon)(r-|y|)^{2 /(m-p)}=M(r) .
$$

2) Допустим теперь, что

$$
u_{0}(x) \geqslant d|x-a|^{2 /(m-p)}
$$

для некоторого $а \in \mathbb{R}^{N} u d>c_{N}, u$ пусть $u(x, t)$ - соответствующее обобщенное решение задачи (1.1), (1.2). Тогда существует положсительная постоянная $k$ такая, что

$$
u(x, t) \geqslant k t^{1 /(1-p)} \quad \text { для любого } \quad x \in \mathbb{R}^{N} \quad u \quad t>0
$$

Теорема 3 может рассматриваться как первый этап описания асимптотического поведения решений задачи (1.1), (1.2). Однако она не дает никакой информации в случае начальных значений с критическим ростом на бесконечности, в частности для

$$
u_{0}(x)=\left[c_{N}|x|^{2 /(m-p)} \pm 1\right]_{+}
$$

Здесь использовано обозначение $s_{+}=\max \{s, 0\}$.

Естественными кандидатами на роли асимптотических состояний являются стационарные решения уравнения (1.1). В некоторых случаях стационарные решения могут быть точно выписаны. Например, для $N=1$ и для некоторых действительных значений $\alpha$ и $\beta$ функции

$$
u_{l}(x ; \alpha)= \begin{cases}c_{1}|x-\alpha|^{2 /(m-p)}, & \text { если } x \leqslant \alpha, \\ 0, & \text { если } x>\alpha,\end{cases}
$$

И

$$
u_{r}(x ; \beta)= \begin{cases}0, & \text { если } x \leqslant \beta, \\ c_{1}|x-\beta|^{2 /(m-p)}, & \text { если } x>\beta,\end{cases}
$$


являются решениями уравнения (1.1). Более того, если $\alpha \leqslant \beta$, то

$$
u(x ; \alpha, \beta)=u_{l}(x ; \alpha)+u_{r}(x ; \beta)
$$

- также решение уравнения (1.1), причем имеюшее на отрезке $[\alpha, \beta]$ "мертвую зону". При $\alpha=\beta=a$ это решение совпадает с одномерным решением из (1.6). Однако существуют стационарные решения (1.1), которые не выписываются в явном виде. Такими являются, например, решения $u_{b}(r)$ следуюшей задачи:

$$
\begin{gathered}
\left(u_{b}^{m}\right)^{\prime \prime}+\frac{N-1}{r}\left(u_{b}^{m}\right)^{\prime}=u_{b}^{p} \text { при } r>0, \quad N \geqslant 1, \\
u_{b}^{m}(0)=b>0, \quad\left(u_{b}^{m}\right)^{\prime}(0)=0 .
\end{gathered}
$$

Перейдем теперь к описанию основных результатов. Для удобства начнем с одномерного случая.

ТЕОрема 4. Предположим, что $u_{0}(x)$ - неотрицательная функция со свойствами, гарантирующими существование обобщенного решения задачи (1.1), (1.2) в $S$.

a) $\Pi$ у $\mathrm{cmb}$

$$
u_{0}(x)= \begin{cases}c_{1}|x|^{\frac{2}{m-p}}+\nu|x|^{\frac{2+p-m}{m-p}}+o\left(|x|^{\frac{2+p-m}{m-p}}\right) & \text { npu } x \leqslant 0 \\ c_{1}|x|^{\frac{2}{m-p}}+h(x) & \text { npu } x>0\end{cases}
$$

әде $\nu$ - некоторое действительное число и

$$
\lim _{x \rightarrow+\infty} h(x)|x|^{-\frac{2+p-m}{m-p}}=-\infty
$$

Тогда

$$
\lim _{t \rightarrow \infty} u(x, t)=u_{l}(x ; \alpha) \quad c \quad \alpha=\frac{(m-p) \nu}{2 c_{1}}
$$

равномерно на компактах $\mathbb{R}$.

b) Предположсим, что

$$
u_{0}(x)= \begin{cases}c_{1}|x|^{\frac{2}{m-p}}+h(x) & \text { nрu } x \leqslant 0 \\ c_{1}|x|^{\frac{2}{m-p}}+\mu|x|^{\frac{2+p-m}{m-p}}+o\left(|x|^{\frac{2+p-m}{m-p}}\right) & \text { npu } x>0\end{cases}
$$

әде $\mu$-некоторое действительное число и

$$
\lim _{x \rightarrow-\infty} h(x)|x|^{-\frac{2+p-m}{m-p}}=-\infty
$$

Тогда

$$
\lim _{t \rightarrow \infty} u(x, t)=u_{r}(x ; \beta) \quad c \quad \beta=-\frac{(m-p) \mu}{2 c_{1}}
$$

равномерно на компактах $\mathbb{R}$.

c) Пусть

$$
u_{0}(x)=c_{1}|x|^{\frac{2}{m-p}}+h(x)
$$


$2 \partial e$

$$
\lim _{|x| \rightarrow \infty} h(x)|x|^{-\frac{2+p-m}{m-p}}=-\infty .
$$

Тогда $\lim _{t \rightarrow \infty} u(x, t)=0$ равномерно накомпактах $\mathbb{R}$.

d) Eсли выполнено $(1.19)$, әде $h(x)|x|^{-\frac{2+p-m}{m-p}} \rightarrow+\infty$ при $x \rightarrow+\infty$ или $x \rightarrow-\infty$, mo $\lim _{t \rightarrow \infty} u(x, t)=+\infty$ равномерно накомпактах $\mathbb{R}$.

е) Допустим, что

$$
u_{0}(x)= \begin{cases}c_{1}|x|^{\frac{2}{m-p}}+\nu|x|^{\frac{2+p-m}{m-p}}+o\left(|x|^{\frac{2+p-m}{m-p}}\right) & \text { npu } x \leqslant 0, \\ c_{1}|x|^{\frac{2}{m-p}}+\mu|x|^{\frac{2+p-m}{m-p}}+o\left(|x|^{\frac{2+p-m}{m-p}}\right) & \text { npu } x>0 .\end{cases}
$$

Тогда появляются два случая:

e1) ecлu $\nu+\mu \leqslant 0, m o$

$$
\lim _{t \rightarrow \infty} u(x, t)=u_{l}(x ; \alpha)+u_{r}(x ; \beta)
$$

равномерно на компактах $\mathbb{R}$, әде $\alpha=(m-p) \nu /\left(2 c_{1}\right)$ и $\beta=-(m-p) \mu /\left(2 c_{1}\right) ;$ е2) если $\nu+\mu>0$, mo

$$
\lim _{t \rightarrow \infty} u(x, t)=u_{b}(x ; a)
$$

равномерно на компактах $\mathbb{R}$, где $u_{b}(x ; a)$ - решение задачи (1.16) $c$ $N=1, r=|x-a|, a=(m-p)(\nu-\mu) /\left(4 c_{1}\right), b=\left[(\nu+\mu) m /\left(2 c_{1}^{1-m} \theta_{1}\right)\right]^{\frac{2 m}{m-p}}$; здесь $\theta_{1}$ - положительная постоянная, зависящая только от $\mathrm{p} / \mathrm{m}$.

ЗАмечАнИЕ 1. Отметим, что условия п. d) теоремы могут выполняться и для функции $u_{0}(x)$, равной нулю на луче.

Если $N>1$, стационарные решения имеют более богатую структуру, и поэтому мы ограничимся радиальным случаем. Тогда результаты похожи на те, которые получены в одномерном случае. Положим

$$
\lambda=\frac{1}{2}\left[\beta_{N}-\left(\beta_{N}^{2}-\frac{4(m-p)}{m} c_{N}^{p-m}\right)^{1 / 2}\right],
$$

где $\beta_{N}=4 m /(m-p)+N-2$, и обозначим через $u(r ; R)$ решение $u_{b}(r)$ из $(1.16)$ с $b=0$ и "мертвой зоной" $B_{R}=\left\{x \in \mathbb{R}^{N}:|x| \leqslant R\right\}$. Легко проверить, что $\lambda<2 m /(m-p), \lambda=1$ при $N=1$ и $\lambda>1$ при $N>1$.

ТЕОРема 5. Предположим, что и ${ }_{0}(r)$ - неотрицательная радиально-симметричная функиия со свойствами, гарантирующими существование обобщенного решения задачи (1.1), (1.2) в S. Тогда обобщенное решение (1.1) с начальным условием $u_{0}(r)$ радиально-симметрично и обладает следующими свойствами.

а) Допустим, что

$$
u_{0}(r)=c_{N} r^{\frac{2}{m-p}}+h(r)
$$

əдe

$$
\lim _{r \rightarrow \infty} h(r) r^{-\left(\frac{2}{m-p}-\lambda\right)}=-\infty .
$$

Тогда $\lim _{t \rightarrow \infty} u(r, t)=0$ равномерно накомпактах $\mathbb{R}^{N}$. 
b) Пусть выполнено (1.24), где

$$
\lim _{r \rightarrow \infty} h(r) r^{-\left(\frac{2}{m-p}-\lambda\right)}=+\infty .
$$

Тогда $\lim _{t \rightarrow \infty} u(r, t)=+\infty$ равномерно на компактах $\mathbb{R}^{N}$.

c) Предположим, что

$$
u_{0}(r)=c_{N} r^{\frac{2}{m-p}}+\nu r^{\frac{2}{m-p}-\lambda}+o\left(r^{\frac{2}{m-p}-\lambda}\right) .
$$

Тогда возникают два случая:

c1) $е с л и \nu \leqslant 0, m o$

$$
\lim _{t \rightarrow \infty} u(r, t)=u(r ; R)
$$

равномерно на компактах $\mathbb{R}^{N}$, әде $R=\left[|\nu| m /\left(c_{N}^{1-m} q_{N}\right)\right]^{1 / \lambda}$ и $q_{N}-$ полодичельная постоянная, зависящая только от $\mathrm{p} / \mathrm{m}$ и $\mathrm{N}$;

с2) если $\nu>0$, mo

$$
\lim _{t \rightarrow \infty} u(r, t)=u_{b}(r)
$$

равномерно на компактах $\mathbb{R}^{N}$, әде $u_{b}(r)$ - решение задачи $(1.16), b=$ $\left[\nu m /\left(c_{N}^{1-m} \theta_{N}\right)\right]^{\frac{2 m}{(m-p) \lambda}}, a \theta_{N}-$ полодсительная постоянная, зависящая только от $\mathrm{p} / \mathrm{m}$ u $N$.

Заметим, что большое количество асимптотических результатов может быть получено для нерадиальных решений, если их начальные значения надлежащим образом оцениваются через соответствуюшие радиальные функции.

Как видно из следуюшего утверждения, у решений, сходящихся к функциям с "мертвыми зонами", такие зоны возникают за конечное время.

Tеорема 6. Пусть для некоторого $R>0$ (может бъть $R=\infty$ )

$$
\lim _{t \rightarrow \infty} u(r, t)=u(r ; R)
$$

равномерно на множестве $B_{R}$. Тогда для любого $d \in(0, R)$ существует положительная постоянная $t_{d}$ такая, что $u(r, t)=0$ для любого $0 \leqslant r \leqslant d$ npu $t \geqslant t_{d}$.

Теорема сформулирована в многомерном случае. Для одномерной ситуации может быть доказано более общее утверждение.

ЗАмЕчАнИЕ 2. Очевидно, предположения теоремы 6 справедливы при выполнении условий пп. а), b), с) и е1) теоремы 4 и пп. а) и с1) теоремы 5.

ЗАмечАниЕ 3 . Как следует из работы [10], при $0<m \leqslant p<1$ уравнение (1.1) обладает бесконечной скоростью распространения возмушений, и, следовательно, обобщенное решение задачи Коши в этом случае может обрашаться в нуль только во всем пространстве одновременно.

Результаты настоящей работы докладывались на конференциях "Нелинейные дифференциальные уравнения с частными производными" в Киеве в 1997 году и "Совместные заседания семинара имени И.Г. Петровского и Московского математического общества" в Москве в 1998 году. 


\section{§ 2. Вспомогательные утверждения}

Формулируемая в настоящем параграфе теорема 7 доказывается так же, как это сделано для ее аналогов в работах [3], [5]-[7].

Теорема 7. Пусть $m>p, m \neq 1 u v(x, t), u(x, t), w(x, t)$-соответственно обобщенные субрешение, решение и суперрешение в $S$ уравнения (1.1), причем

$$
v(x, 0) \leqslant u(x, 0) \leqslant w(x, 0) .
$$

$B$ случае $m>1$ дополнительно предположсим, что функиии $v(x, t), u(x, t)$ и $w(x, t)$ принадлежсат классу $K$. Тогда в $S$

$$
v(x, t) \leqslant u(x, t) \leqslant w(x, t) .
$$

В дальнейшем нам понадобятся некоторые сведения о стационарных решениях уравнения (1.1). Следующие два утверждения доказаны в [2]. Пусть $u_{b}(x)$ - решение задачи (1.16) при $x \in \mathbb{R}$ и $b \geqslant 0$.

ПРЕДЛОЖЕНИЕ 1. Предположим, что $N=1$.

а) Пусть $b>0$. Тогда существует единственное решение задачи (1.16). Более того,

$$
u_{b}^{m}(x)=c_{1}^{m}|x|^{\frac{2 m}{m-p}}+\theta_{1} b^{\frac{m-p}{2 m}}|x|^{\frac{m+p}{m-p}}+o\left(|x|^{\frac{m+p}{m-p}}\right),
$$

әде постоянная $c_{1}$ определена в (1.6), а $\theta_{1}$ - положительная постоянная, зависяицая только от $\mathrm{p} / \mathrm{m}$.

b) Предположим, что $b=0$. Тогда любое нетривиальное решение $u_{b}(x)$ является одной из перечисленных ниже функиий:

$$
\begin{gathered}
u_{l}(x ; \alpha) \text { для некоторого } \alpha, \quad u_{r}(x ; \beta) \text { для некоторого } \beta \quad \text { или } \\
u_{l}(x ; \alpha)+u_{r}(x ; \beta) \text { для некоторьх } \alpha \text { и } \beta \text { таких, что } \alpha \leqslant \beta .
\end{gathered}
$$

Функиии $u_{l}(x ; \alpha)$ и $u_{r}(x ; \beta)$ определеньв в (1.13) и (1.14).

Пусть теперь $u_{b}(r)$ - решение задачи (1.16) с $N>1$ и $b \geqslant 0$.

ПРЕДЛОЖЕНИЕ 2. Предположим, что $N>1$.

а) Пусть $b>0$. Тогда существует единственное решение $u_{b}(r)$ задачи (1.16), определенное для любого $r>0$. Более того,

$$
u_{b}^{m}(r)=c_{N}^{m} r^{\frac{2 m}{m-p}}+\theta_{N} b^{\frac{m-p}{2 m} \lambda} r^{\frac{2 m}{m-p}-\lambda}+o\left(r^{\frac{2 m}{m-p}-\lambda}\right)
$$

где $c_{N}$ определена в (1.6), $\theta_{N}$ - положстельная постоянная, зависящая только от $p / m$ и $N, \lambda$ - постоянная из (1.23).

b) Пусть $b=0$. Тогда для любого $R \geqslant 0$ существует единственное решение $u(r ; R)$ задачи (1.16) такое, что $u(r ; R)=0$ при $r \leqslant R u u(r ; R)>0$ nри $r>R$. Более того,

$$
u^{m}(r ; R)=c_{N}^{m} r^{\frac{2 m}{m-p}}-q_{N} R^{\lambda} r^{\frac{2 m}{m-p}-\lambda}+o\left(r^{\frac{2 m}{m-p}-\lambda}\right)
$$

для некоторой положительной постоянной $q_{N}$, зависящей только от $N$ $u p / m$.

Все нетривиальные решения задачи (1.16) принадлежсат одному из двух перечисленных выше типов. 
ЗАмЕЧАнИЕ 4. Отметим, что не сушествует различных стационарных решений $u$ и $v$ уравнения (1.1) из предложений 1 и 2 таких, чтобы разность $u^{m}-v^{m}$ была ограниченной функцией.

Лемма 1. Для любого $R \geqslant 0$ справедливо следующее неравенство:

$$
u(r ; R) \geqslant c_{N}(r-R)_{+}^{\frac{2}{m-p}} .
$$

ДокАЗАТЕЛЬство. Очевидно, при $R=0$ неравенство в (2.4) превращается в равенство. Пусть $R>0$. Как следует из предложения 2 , функция $v(r)=u^{m}(r ; R)$ является единственным положительньм при $r>R$ решением задачи

$$
\begin{gathered}
L v \equiv v^{\prime \prime}+\frac{N-1}{r} v^{\prime}-v^{p / m}=0, \quad r>R, \\
v(R)=0, \quad v^{\prime}(R)=0 .
\end{gathered}
$$

Покажем, что $v^{\prime}(r)>0$ при $r>R$. Допустим, что сушествует значение $r_{0}>R$, для которого $v^{\prime}\left(r_{0}\right)=0$. Тогда так как $v(r)>0$ при $r>R$, то $v^{\prime \prime}\left(r_{0}\right)>0$. Следовательно, $r_{0}$ - точка минимума $v(r)$. Но тогда найдется значение $r_{1} \in\left(R, r_{0}\right)$, при котором функция $v(r)$ имеет максимум. Из уравнения (2.5) находим, что $v^{\prime \prime}\left(r_{1}\right)>0$, и возникает противоречие. Определим для любого $\varepsilon>0$ функцию $h(r)=c_{N}^{m}(r-R-\varepsilon)_{+}^{2 m /(m-p)}$ и сравним ее при $r \geqslant R$ с $v(r)$. Несложно проверить, что $L h<0$ при $r>R+\varepsilon$. Вследствие доказанных свойств функции $v(r)$ для некоторого $R_{0}>R+\varepsilon v^{\prime}>h^{\prime}$ и $v>h$ при $r \in\left(R+\varepsilon, R_{0}\right)$. Предположим, что найдется такое значение $R_{1}>R+\varepsilon$, что $v^{\prime}\left(R_{1}\right)=h^{\prime}\left(R_{1}\right)$ и $v^{\prime}(r)>h^{\prime}(r)$ для $r \in\left(R+\varepsilon, R_{1}\right)$. Легко видеть, что $v>h$ при $r \in\left(R+\varepsilon, R_{1}\right]$. Тогда в точке $r=R_{1}$

$$
(v-h)^{\prime \prime}>v^{p / m}-h^{p / m}>0
$$

что противоречит условию $v^{\prime}\left(R_{1}\right)=h^{\prime}\left(R_{1}\right)$. Следовательно, $v(r)>h(r)$ при $r>R$. В силу произвольности $\varepsilon$ отсюда вытекает (2.4).

ЗАмечАнИЕ 5. Отметим, что радиально-симметричные стационарные решения уравнения (1.1) являются неубьвающими функциями переменной $r$.

\section{§ 3. Доказательство теоремы 3}

Начнем с доказательства п. 1). Без ограничения общности можно считать, что $a=0$. Зафиксируем произвольное значение $y \in \mathbb{R}^{N}$ и покажем, что в предположениях $(1.7),(1.8)$ существует такое значение $T_{E}(y)$, что $u(y, t)=0$ при $t \geqslant T_{E}(y)$.

Рассмотрим сначала случай $m>1$. Выберем числа $\varepsilon$ и $\varepsilon_{1}$ так, чтобы выполнялись соотношения

$$
0<\varepsilon<c_{N}-B, \quad \varepsilon_{1}=1-\left(\frac{B+\varepsilon}{c_{N}}\right)^{m},
$$

где постоянные $c_{N}$ и $B$ удовлетворяют $(1.6),(1.8)$. Определим вспомогательную функцию $w(x, t)$ равенством

$$
w(x, t)=\left\{\varepsilon_{1} g^{m}(t)+\left(1-\varepsilon_{1}\right) u^{m}(r ;|y|)\right\}^{1 / m},
$$


где $g(t)=\left(K-\varepsilon_{1}^{(m-1) / m}(1-p) t\right)_{+}^{1 /(1-p)}, u(r,|y|)-$ решение задачи $(1.16)$, положительное при $r>|y|$ и равное нулю при $r \leqslant|y|, K$ - положительная постоянная, которая будет определена ниже. Используя свойство выпуклости функции $z(s)=s^{p / m}$, получаем

$$
w^{p} \geqslant \varepsilon_{1} g^{p}(t)+\left(1-\varepsilon_{1}\right) u^{p}(r ;|y|)
$$

Отсюда и из определения функции $w(x, t)$ находим, что

$$
w_{t}-\Delta w^{m}+w^{p} \geqslant 0
$$

Займемся теперь выбором значения $K$, при котором бы выполнялось неравенство

$$
u_{0}(x) \leqslant w(x, 0)
$$

В силу (3.1) и (2.4)

$$
(B+\varepsilon)(r-|y|)_{+}^{2 /(m-p)} \leqslant \frac{B+\varepsilon}{c_{N}} u(r ;|y|)=\left(1-\varepsilon_{1}\right)^{1 / m} u(r ;|y|)
$$

Вследствие (1.7) значение $R=R(\varepsilon)$ из формулировки теоремы корректно определено. Из (3.2) и (3.6) находим, что (3.5) выполнено при $|x| \geqslant R$. Для того чтобы $(3.5)$ было справедливо и при $|x|<R$, достаточно выполнения равенства

$$
\varepsilon_{1}^{1 / m} g(0)=M(R)
$$

Отсюда $K=\left[M(R) \varepsilon_{1}^{-1 / m}\right]^{1-p}$. По теореме 7 из $(3.4),(3.5)$ выводим оценку

$$
u(x, t) \leqslant w(x, t)
$$

Но в силу $(3.2) w(y, t)=0$ при $t \geqslant T(y)=K /\left[(1-p) \varepsilon_{1}^{(m-1) / m}\right]$. Подставляя сюда значение для $K$ из $(3.7)$ и $\varepsilon_{1}$ из $(3.1)$, получаем

$$
T(y)=\frac{1}{1-p} \frac{[M(R(\varepsilon))]^{1-p}}{\left[1-\left((B+\varepsilon) / c_{N}\right)^{m}\right]^{\frac{m-p}{m}}}
$$

откуда следует оценка (1.9). 
Доказательство в случае $p<m<1$ проводится аналогичным образом. Укажем лишь на те изменения, которые необходимо внести в доказательство. В (3.1) $\varepsilon_{1}$ следует определить формулой

$$
\varepsilon_{1}=1-\left(\frac{B+\varepsilon}{c_{N}}\right)^{\frac{m-p}{1-p}}
$$

а в (3.2) вместо $w(x, t)$ рассмотреть вспомогательную функцию

$$
z(x, t)=\varepsilon_{1} g_{1}(t)+\left(1-\varepsilon_{1}\right)^{\frac{1-p}{m-p}} u(r ;|y|),
$$

где $g_{1}(t)=(K-(1-p) t)_{+}^{\frac{1}{1-p}}$. Правая часть оценки $(3.6)$ заменится на выражение $\left(1-\varepsilon_{1}\right)^{\frac{1-p}{m-p}} u(r ;|y|)$, а $(3.7)$ на соотношение $\varepsilon_{1} g_{1}(0)=M(R)$.

Установим теперь справедливость п. 2) теоремы 3. Как и при доказательстве первой части теоремы, будем считать, что $a=0$. Рассмотрим задачу Коши $(1.1),(1.2) \mathrm{c}$

$$
u_{0}(x)=\psi_{c}(x)=c|x|^{\frac{2}{m-p}} .
$$

Как легко видеть из теорем 1 и 2 , эта задача имеет единственное радиально-симметричное решение $u_{c}(x, t)$. Положим $\alpha=1 /(1-p), \beta=\frac{1}{2}(m-p) /(1-p)$.

Лемма 2. Задача (1.1), (3.9) при $c>c_{N}$ имеет автомодельное решение

$$
u_{c}(x, t)=t^{\alpha} f_{c}(\eta)
$$

әде $\eta=|x| t^{-\beta}$, а функиия $f_{c}(\eta)$ является решением задачи

$$
\begin{gathered}
\left(f^{m}\right)^{\prime \prime}+\frac{N-1}{\eta}\left(f^{m}\right)^{\prime}+\beta \eta f^{\prime}-\alpha f-f^{p}=0, \quad \eta>0 \\
f^{m}(0)=A \geqslant 0, \quad\left(f^{m}\right)^{\prime}(0)=0
\end{gathered}
$$

и удовлетворяет соотношениям

$$
f_{c}(\eta)>0 \quad n p u \quad \eta>0, \quad \lim _{\eta \rightarrow+\infty} \frac{f_{c}(\eta)}{\eta^{\frac{2}{m-p}}}=c .
$$

ДокАЗАТЕЛЬство. Покажем, что функция $u_{c}(x, t)$ является автомодельным решением уравнения (1.1). Преобразование $u \rightarrow P_{k} u$, определенное при $k>0$ по формуле

$$
\left(P_{k} u\right)(x, t)=k^{-\frac{2}{m-p}} u\left(k x, k^{\frac{2(1-p)}{m-p}} t\right)
$$

переводит решения уравнения (1.1) снова в решения уравнения (1.1). Заметим также, что $\psi_{c}(x)$ инвариантна относительно этого преобразования:

$$
k^{-\frac{2}{m-p}} \psi_{c}(k x)=\psi_{c}(x) .
$$


В силу равенства (3.13) и единственности решения задачи (1.1), (3.9) получаем, что $P_{k} u_{c}=u_{c}$ или

$$
u_{c}(x, t)=k^{-\frac{2}{m-p}} u_{c}\left(k x, k^{\frac{2(1-p)}{m-p}} t\right) .
$$

Положим здесь $t=1, k^{\frac{2(1-p)}{m-p}}=s$ и $k x=y$. Тогда

$$
u_{c}\left(s^{-\frac{m-p}{2(1-p)}} y, 1\right)=s^{-\frac{1}{1-p}} u_{c}(y, s) .
$$

Заменяя $y$ и $s$ на $x$ и $t$ соответственно и умножая затем равенство на $t^{\frac{1}{1-p}}$, получаем

$$
u_{c}(x, t)=t^{\frac{1}{1-p}} \varphi_{c}(\xi)
$$

где $\xi=x t^{-\frac{m-p}{2(1-p)}}, \varphi_{c}(\xi)=u_{c}(\xi, 1)$. По теореме 7 при $c>c_{N} u_{c}(x, t) \geqslant u_{c_{N}}(x, t)$. Отсюда, учитьвая, что $\varphi_{c_{N}}=c_{N}|\xi|^{\frac{2}{m-p}}$, находим

$$
\varphi_{c}(\xi) \geqslant c_{N}|\xi|^{\frac{2}{m-p}} .
$$

В частности, $\varphi_{c}(\xi)>0$ при $\xi \neq 0$. В тех точках $\xi$, где $\varphi(\xi)>0$, функция $u_{c}(x, t)$, как известно, удовлетворяет уравнению в обычном смысле. Подставляя функцию из (3.14) в (1.1) и учитывая радиальную симметричность $u_{c}(x, t)$, получаем для $f_{c}(\eta)=\varphi_{c}(\xi), \eta=|\xi|$ задачу (3.11). Очевидно, $f_{c}(\eta)>0$ при $\eta>0$. Так как для любого $x \in \mathbb{R}^{N}$ выполняется начальное условие

$$
\lim _{t \rightarrow 0} u_{c}(x, t)=\lim _{t \rightarrow 0} t^{\frac{1}{1-p}} f_{c}(\eta)=c|x|^{\frac{2}{m-p}},
$$

то справедливо второе соотношение в (3.12).

ЛЕмма 3. При $c>c_{N}$ функиия $f_{c}(\eta)$ является решением задачи (3.11) с $A>0$.

ДокАЗАтЕльство. Предположим сначала, что $m+p<2$. Покажем, что $u_{c}(0, t)>0$ при достаточно малых положительных значениях $t$. Это можно сделать, сравнивая $u_{c}(x, t)$ с функцией $w(x, t)=d_{1}(|x|+\gamma t)^{\frac{2}{m-p}}$ в цилиндре $Q_{\delta}=$ $\left\{(x, t):|x| \leqslant \delta, 0 \leqslant t \leqslant t_{\delta}\right\}$. Здесь $c_{N}<d_{1}<c$. При достаточно малых положительных значениях $\gamma, \delta$ и $t_{\delta}$ функция $w(x, t)$ является обобшенным субрешением уравнения (1.1) и на параболической границе $Q_{\delta}$ выполняется неравенство $u_{c}(x, t) \geqslant w(x, t)$. По теореме сравнения это неравенство справедливо во всем $Q_{\delta}$. Следовательно, при $0<t \leqslant t_{\delta} \quad u_{c}(0, t) \geqslant w(0, t)>0$. В силу представления (3.10) $f_{c}(0)>0$.

Осталось доказать утверждение леммы для случая $m+p \geqslant 2$. Для удобства сделаем в (3.11) замену $v=f_{c}^{m}$. Тогда функция $v(\eta)$ является решением задачи

$$
\begin{gathered}
L v \equiv v^{\prime \prime}+\frac{N-1}{\eta} v^{\prime}+\beta \eta\left(v^{1 / m}\right)^{\prime}-\alpha v^{1 / m}-v^{p / m}=0, \quad \eta>0, \\
v(0)=A, \quad v^{\prime}(0)=0 .
\end{gathered}
$$

Аналогично тому, как это сделано в [11], доказывается следующее утверждение. 
ПРЕДЛОЖЕНИЕ 3. Пусть $L(h)>L(v) n p u \eta>0 u h(0) \geqslant v(0), h^{\prime}(0)>v^{\prime}(0)$. Тогда $h(\eta)>v(\eta)$ при $\eta>0$.

Предположим, что в (3.15) $A=0$, и получим противоречие. Для этого сравним с помощью предложения 3 функции $v(\eta)$ и $h(\eta)=c^{m}(\eta+\varepsilon)^{\frac{2 m}{m-p}}$, где $c>c_{N}$, а постоянная $\varepsilon>0$ будет выбрана ниже. Непосредственной проверкой убеждаемся в том, что $h(0)>0, h^{\prime}(0)>0$ и $L h>0$ при

$$
0<\varepsilon<\left\{\frac{1}{\alpha c} c_{N}^{p}\left[\left(\frac{c}{c_{N}}\right)^{m}-\left(\frac{c}{c_{N}}\right)^{p}\right]\right\}^{\frac{m-p}{2(1-p)}} .
$$

Таким образом, любое решение задачи (3.15) с $A=0$ удовлетворяет неравенству

$$
v(\eta) \leqslant c^{m}(\eta+\varepsilon)^{\frac{2 m}{m-p}},
$$

где $c$ - произвольная постоянная, большая, чем $c_{N}$, а $\varepsilon$ удовлетворяет (3.16). Отсюда приходим к оценке

$$
f_{c}(\eta) \leqslant c_{N} \eta^{\frac{2}{m-p}}
$$

которая противоречит (3.12). Легко доказьвается

Лемма 4. Пусть в (3.11) $A>0$. Тогда решение $f_{c}(\eta)$ этой задачи удовлетворяет неравенствам

$$
f_{c}(\eta)>A, \quad f_{c}^{\prime}(\eta)>0 \quad \text { nрu } \quad \eta>0 .
$$

Теперь несложно завершить доказательство п. 2). Действительно, сравним функции $u(x, t)$ и $u_{c}(x, t) \mathrm{c} c=d>c_{N}$. В силу (1.11) и теоремы 7

$$
u(x, t) \geqslant u_{c}(x, t) .
$$

Вследствие (3.10) и лемм 3 и 4

$$
u_{c}(x, t) \geqslant A^{1 / m} t^{\alpha}
$$

с $A>0$. Из (3.18), (3.19) получаем искомое неравенство (1.12).

ЗАмЕчАниЕ 6 . Покажем, что теорема 3 является в определенном смысле точной. Прежде всего, легко видеть, что правые части в неравенствах (1.9) и (1.10) совпадают при $m=1$. Далее, пусть $u_{0}(x) \equiv M>0$. Тогда $M(R)=M$, а в $(1.7)$ можно положить $B=0$. Из (1.9) и (1.10) вытекает оценка $T_{E}(y) \leqslant M^{1-p} /(1-p)$, которая согласуется с точным пространственно-однородньм решением задачи Кошши $u(x, t)=\left[M^{1-p}-(1-p) t\right]^{\frac{1}{1-p}}$.

Рассмотрим теперь задачу Коши $(1.1),(3.9) \mathrm{c} c<c_{N}$. Решение $u_{c}(x, t)$ этой задачи находится по формуле (3.10). Из сравнения $u_{c}(x, t)$ с $u_{c_{N}}(x, t)$ следует, что в (3.11) $A=0$. Далее, из первой части теоремы 3 вытекает, что $f_{c}(\eta)=0$ при положительных значениях $\eta_{2}=\eta_{2}(c)$ и $\varepsilon$. Нетрудно установить, что $f_{c}(\eta)>0$ при $\eta>\eta_{2}$. Таким образом, из (3.10) для любого $y \in \mathbb{R}^{N}$

$$
T_{E}(y)=\left[\frac{|y|}{\eta_{2}}\right]^{\frac{2(1-p)}{m-p}} .
$$


Определим теперь $T_{E}(y)$ из теоремы 3. Используя определение $R(\varepsilon)$ с $B=c$, находим

$$
R(\varepsilon)=\frac{|y|}{1-(c /(c+\varepsilon))^{\frac{m-p}{2}}} .
$$

Подставляя теперь найденное значение $R(\varepsilon)$ в правые части оценок $(1.9)$ и (1.10), приходим к неравенству

$$
T_{E}(y) \leqslant d_{2}|y|^{\frac{2(1-p)}{m-p}},
$$

где положительная постоянная $d_{2}$ зависит от $m, p, N$ и $c$. Очевидно, оценка (3.21) согласуется с найденным в (3.20) значением времени "остывания" обобщенного решения задачи $(1.1),(3.9)$.

Если рассмотреть обобшенное решение задачи (1.1), (3.9) с $c>c_{N}$, то, как видно из $(3.10),(3.11), u_{c}(0, t)=A^{1 / m} t^{\alpha}$ и

$$
\lim _{t \rightarrow \infty} \frac{u_{c}(x, t)}{t^{\alpha}}=A^{1 / m}>0
$$

для любого $x \in \mathbb{R}^{N}$. Соотношение (3.22) показывает точность (1.12) и подтверждает независимость $k$ от $x$.

\section{§4. Доказательство основных результатов}

Пусть $u_{s}(r)$ - радиально-симметричное стационарное решение уравнения (1.1). Обозначим соответственно через $\bar{u}(r, t)$ и $\underline{u}(r, t)$ обобшенные решения задач Коши с начальными условиями

$$
\bar{u}(r, 0)=\left(u_{s}^{m}(r)+M\right)^{1 / m}, \quad \underline{u}(r, 0)=\left(u_{s}^{m}(r)-M\right)_{+}^{1 / m} .
$$

где $M$ - некоторая положительная постоянная. Докажем асимптотическую устойчивость стационарных решений относительно возмущений начальной функции вида (4.1).

ЛЕмма 5. Справедливъ соотноиения

$$
\lim _{t \rightarrow \infty} \bar{u}(r, t)=u_{s}(r), \quad \lim _{t \rightarrow \infty} \underline{u}(r, t)=u_{s}(r)
$$

равномерно на любом компактном подмножестве $\mathbb{R}^{N}$.

ДокАЗАтельство. Докажем сначала первое равенство в (4.2). Для этого установим монотонность функции $\bar{u}(r, t)$ по переменной $t$. Несложно проверить, что $u_{s}(r)$ и $u_{M}(r)=\left[u_{s}^{m}(r)+M\right]^{1 / m}$ являются соответственно решением и суперрешением уравнения (1.1), причем

$$
u_{s}(r)<\bar{u}(r, 0)=u_{M}(r)
$$

По теореме 7 получаем

$$
u_{s}(r) \leqslant \bar{u}(r, t) \leqslant u_{M}(r)
$$

В частности, $\bar{u}(r, t) \leqslant \bar{u}(r, 0)$. Очевидно, функция $\bar{u}(r, t+h)$ для любого положительного значения $h$ является обобщенным решением $(1.1)$ и $\bar{u}(r, h) \leqslant \bar{u}(r, 0)$. 
Применяя опять теорему 7 , находим $\bar{u}(r, t+h) \leqslant \bar{u}(r, t)$, что доказывает монотонное невозрастание $\bar{u}(r, t)$ по переменной $t$. Отсюда с использованием (4.3) делаем вьвод, что существует локально ограниченная функция $\varphi(r)$ такая, что

$$
\begin{gathered}
\lim _{t \rightarrow \infty} \bar{u}(r, t)=\varphi(r), \\
u_{s}(r) \leqslant \varphi(r) \leqslant\left[u_{s}^{m}(r)+M\right]^{1 / m} .
\end{gathered}
$$

Для любого $a \geqslant 0$ определим функцию $w_{a}(r, t)$ равенством $w_{a}(r, t)=\bar{u}(r, t+a)$. Легко проверить, что $w_{a}(r, t)$ является обобшенным решением уравнения (1.1) и удовлетворяет начальному условию $w_{a}(r, 0)=\bar{u}(r, a)$. С другой стороны, однопараметрическое семейство функций $\left\{w_{a}(r, t)\right\}$ монотонно не возрастает по $a$. Поэтому функция $\theta(r, t)$ корректно определена равенством

$$
\theta(r, t)=\lim _{a \rightarrow \infty} w_{a}(r, t)
$$

С помощью стандартных рассуждений проверяем, что $\theta(r, t)$ - обобщенное решение уравнения (1.1). В силу (4.4), (4.6) $\varphi(r)=\theta(r, t)$ и, следовательно, $\varphi(r)-$ обобшенное стационарное решение (1.1), удовлетворяющее (4.5). Как отмечено в замечании 4 , не сушествует двух различных радиально-симметричных стационарных решений $h(r)$ и $g(r)$ уравнения $(1.1)$, для которых разность $h^{m}(r)-g^{m}(r)$ была бы ограниченной функцией. Значит, $\varphi(r)=u_{s}(r)$ и мы имеем поточечную сходимость $\bar{u}(r, t)$ к $u_{s}(r)$ при $t \rightarrow \infty$. Докажем теперь равномерность этой сходимости на любом ограниченном множестве $\mathbb{R}^{N}$. Пусть $\left\{t_{n}\right\}-$ произвольная монотонно возрастающая последовательность положительных чисел такая, что $t_{n} \rightarrow \infty$ при $n \rightarrow \infty$. Обозначим $\bar{u}_{n}(r)=\bar{u}\left(r, t_{n}\right)$ и рассмотрим последовательность $\left\{\bar{u}_{n}(r)\right\}$ в произвольном шаре $B_{R}$. Очевидно, $\bar{u}_{n+1}(r) \leqslant \bar{u}_{n}(r)$ для всех $n \geqslant 1$ и $\lim _{n \rightarrow \infty} \bar{u}_{n}(r)=u_{s}(r)$. Но отсюда согласно признаку Дини равномерной сходимости функциональных последовательностей сходимость $\bar{u}_{n}(r)$ к $u_{s}(r)$ равномерная в $B_{R}$. В силу монотонности $\bar{u}(r, t)$ по $t$ и сходимость $\bar{u}(r, t)$ к $u_{s}(r)$ равномерная в $B_{R}$.

Второе равенство в (4.2) доказывается аналогично. Отметим, что при доказательстве соответствующего (4.3) неравенства

$$
\left[u_{s}^{m}(r)-M\right]_{+}^{1 / m} \leqslant \underline{u}(r, t) \leqslant u_{s}(r)
$$

используется тот факт, что функции $\left[u_{s}^{m}(r)-M\right]_{+}^{1 / m}$ и $\underline{u}(r, t)$ являются соответственно суб- и суперрешениями первой краевой задачи в $Q_{r_{0}}=\left(\mathbb{R}^{N} \backslash B_{r_{0}}\right) \times \mathbb{R}_{+}$ для уравнения (1.1) с условиями

$$
\begin{gathered}
u(r, 0)=\left[u_{s}^{m}(r)-M\right]_{+}^{1 / m}, \quad r \geqslant r_{0}, \\
u\left(r_{0}, t\right)=0, \quad t \geqslant 0 .
\end{gathered}
$$

Здесь через $r_{0}$ обозначен корень уравнения $u_{s}^{m}(r)=M$. В случае, если это уравнение не имеет корней, сравнивать функции следует во всем пространстве $S$. 
ЗАмЕчАниЕ 7. В одномерном случае аналогичное лемме 5 утверждение об асимптотической устойчивости всех стационарных решений доказывается таким же образом.

ДОКАЗАТЕЛЬСТВо ТЕОРЕМЫ 4. а) Из определения (1.13) функции $u_{l}(x ; \alpha)$ при $x<\min (0, \alpha)$ находим, что

$$
\begin{aligned}
u_{l}(x ; \alpha) & =c_{1}|x-\alpha|^{\frac{2}{m-p}}=c_{1}|x|^{\frac{2}{m-p}}\left(1+\frac{\alpha}{|x|}\right)^{\frac{2}{m-p}} \\
& =c_{1}|x|^{\frac{2}{m-p}}\left(1+\frac{2}{m-p} \frac{\alpha}{|x|}+o\left(\frac{1}{|x|}\right)\right) \\
& =c_{1}|x|^{\frac{2}{m-p}}+\nu|x|^{\frac{2+p-m}{m-p}}+o\left(|x|^{\frac{2+p-m}{m-p}}\right) .
\end{aligned}
$$

В силу предположений относительно $u_{0}(x)$ имеем

$$
\left[u_{l}^{m}(x, \alpha-\varepsilon)-M\right]_{+}^{1 / m} \leqslant u_{0}(x) \leqslant\left\{\left[u_{l}(x, \alpha+\varepsilon)+u_{r}\left(x, \alpha+\frac{1}{\varepsilon}\right)\right]^{m}+M\right\}^{1 / m}
$$

при любом $\varepsilon \in(0,1)$ и достаточно большом значении $M . \mathrm{C}$ помошью теоремы 7 и замечания 7 отсюда выводим, что

$$
u_{l}(x, \alpha-\varepsilon) \leqslant \lim _{t \rightarrow \infty} \inf u(x, t) \leqslant \lim _{t \rightarrow \infty} \sup u(x, t) \leqslant u_{l}(x, \alpha+\varepsilon)+u_{r}\left(x, \alpha+\frac{1}{\varepsilon}\right) .
$$

Устремляя здесь $\varepsilon$ к нулю, получаем (1.17).

Таким же образом устанавливается справедливость п. b).

c) Используя условия теоремы, равенство (4.7) и аналогичное выражение для $u_{r}(x ; \beta)$, получаем, что для любых значений $\alpha$ и $\beta(\alpha<\beta)$ при подходящем выборе постоянной $M$ выполнено неравенство

$$
u_{0}(x) \leqslant\left\{\left[u_{l}(x ; \alpha)+u_{r}(x ; \beta)\right]^{m}+M\right\}^{1 / m} .
$$

Отсюда и из теоремы 7 и замечания 7 следует оценка

$$
\lim _{t \rightarrow \infty} \sup u(x, t) \leqslant u_{l}(x ; \alpha)+u_{r}(x ; \beta)
$$

Вследствие произвольности $[\alpha, \beta]$ из этого неравенства вытекает искомое соотношение.

d) Предположим, например, что для функции $h(x)$ справедливо равенство

$$
\lim _{x \rightarrow+\infty} h(x)|x|^{-\frac{2+p-m}{m-p}}=+\infty
$$

Тогда для любого значения $\sigma>0$ можно построить такую функцию

$$
\varphi(x)= \begin{cases}c_{1}|x|^{\frac{2}{m-p}}+y(x) & \text { при } x \leqslant 0, \\ c_{1}|x|^{\frac{2}{m-p}}+\sigma|x|^{\frac{2+p-m}{m-p}}+o\left(|x|^{\frac{2+p-m}{m-p}}\right) & \text { при } x>0\end{cases}
$$


что $u_{0}(x) \geqslant \varphi(x)$. Здесь $y(x)-$ некоторая функция, для которой

$$
\lim _{x \rightarrow-\infty} y(x)|x|^{-\frac{2+p-m}{m-p}}=-\infty .
$$

Согласно п. b) решение $u_{\varphi}(x, t)$ задачи Коши $(1.1),(1.2)$ с начальным условием $\varphi(x)$ удовлетворяет соотношению

$$
\lim _{t \rightarrow \infty} u_{\varphi}(x, t)=u_{r}(x ; \beta),
$$

где $\beta=-(m-p) \sigma /\left(2 c_{1}\right)$. С другой стороны, по теореме $7 u(x, t) \geqslant u_{\varphi}(x, t)$. Таким образом,

$$
\liminf _{t \rightarrow \infty} u(x, t) \geqslant u_{r}(x ; \beta) .
$$

В силу того что $\beta \rightarrow-\infty$ при $\sigma \rightarrow+\infty$, отсюда вытекает необходимьй результат.

е) Пусть $\nu+\mu<0$. Заметим, что при $\alpha<\beta$

$$
u_{l}(x ; \alpha)+u_{r}(x ; \beta)=\left\{\begin{array}{l}
c_{1}|x|^{\frac{2}{m-p}}+\frac{2 \alpha c_{1}}{m-p}|x|^{\frac{2+p-m}{m-p}}+o\left(|x|^{\frac{2+p-m}{m-p}}\right) \text { при } x \leqslant 0, \\
c_{1}|x|^{\frac{2}{m-p}}-\frac{2 \beta c_{1}}{m-p}|x|^{\frac{2+p-m}{m-p}}+o\left(|x|^{\frac{2+p-m}{m-p}}\right) \text { при } x>0 .
\end{array}\right.
$$

Если положить

$$
\frac{2 \alpha c_{1}}{m-p}=\nu, \quad-\frac{2 \beta c_{1}}{m-p}=\mu,
$$

то условие $\alpha<\beta$ равносильно неравенству $\nu+\mu<0$ и правая часть (1.20) совпадет с правой частью (4.8). Следовательно, для любого достаточно малого $\varepsilon>0$ и подходящего значения $M$

$$
\begin{aligned}
& \left\{\left[u_{l}(x, \alpha-\varepsilon)+u_{r}(x, \beta+\varepsilon)\right]^{m}-M\right\}_{+}^{1 / m} \leqslant u_{0}(x) \\
& \leqslant\left\{\left[u_{l}(x, \alpha+\varepsilon)+u_{r}(x, \beta-\varepsilon)\right]^{m}+M\right\}^{1 / m} .
\end{aligned}
$$

Далее, используя теорему 7 и замечание 7 , получаем (1.21). В случае $\mu+\nu=0$ справедливы неравенства (4.9), где $\alpha=\beta=x_{0}$ и правая часть заменена на выражение $\left\{u_{\varepsilon}^{m}\left(x ; x_{0}\right)+M\right\}^{1 / m}$. Как и раньше, эти оценки приводят к искомому результату.

Пусть теперь $\mu+\nu>0$. Из предложения 1 находим, что

$$
u_{b}(x ; a)=c_{1}|x-a|^{\frac{2}{m-p}}+\frac{1}{m} \theta_{1} b^{\frac{m-p}{2}} c_{1}^{1-m}|x-a|^{\frac{2+p-m}{m-p}}+o\left(|x-a|^{\frac{2+p-m}{m-p}}\right) .
$$

Отсюда следует, что для $u_{b}(x ; a)$ выполнено $(1.20)$ с

откуда

$$
\nu=\frac{1}{m} \theta_{1} b^{\frac{m-p}{2}} c_{1}^{1-m}+\frac{2 a c_{1}}{m-p}, \quad \mu=\frac{1}{m} \theta_{1} b^{\frac{m-p}{2}} c_{1}^{1-m}-\frac{2 a c_{1}}{m-p},
$$

$$
b=\left\{\frac{m(\nu+\mu)}{2 \theta_{1} c_{1}^{1-m}}\right\}^{\frac{2}{m-p}}, \quad a=\frac{(m-p)(\nu-\mu)}{4 c_{1}} .
$$

Таким образом, для любого достаточно малого значения $\varepsilon>0$ сушествует такая положительная постоянная $M$, что справедливы неравенства

$$
\left\{u_{b-\varepsilon}^{m}(x ; a)-M\right\}_{+}^{1 / m} \leqslant u_{0}(x) \leqslant\left\{u_{b+\varepsilon}^{m}(x ; a)+M\right\}^{1 / m} .
$$

Далее с помощью рассуждений, аналогичных предыдущим, доказываем (1.22). 
ДоКАЗАТЕЛЬСТВо ТЕОРЕМЫ 5 . То, что решение, соответствующее радиально-симметричным начальным условиям, является также радиально-симметричным, следует из теоремы существования и единственности. Из предложения 2 выводим, что при $b>0$ для решения $u_{b}(r)$ задачи (1.16) справедливо представление

$$
u_{b}(r)=c_{N} r^{\frac{2}{m-p}}+\frac{1}{m} \theta_{N} b^{\frac{m-p}{2} \lambda} c_{N}^{1-m} r^{\frac{2}{m-p}-\lambda}+o\left(r^{\frac{2}{m-p}-\lambda}\right) .
$$

При $b=0$ для решения $u(r ; R)$ вьполнено соотношение

$$
u(r ; R)=c_{N} r^{\frac{2}{m-p}}-\frac{1}{m} q_{N} R^{\lambda} c_{N}^{1-m} r^{\frac{2}{m-p}-\lambda}+o\left(r^{\frac{2}{m-p}-\lambda}\right) .
$$

Дальнейшее доказательство аналогично приведенному в теореме 4 , и поэтому укажем лишь на то, с какими функциями следует сравнивать начальное условие, чтобы получить требуемый результат. Если выполнены условия (1.24), (1.25) теоремы, то для любого $R>0$ сушествует такая положительная постоянная $M$, что

$$
0 \leqslant u_{0}(r) \leqslant\left[u^{m}(r ; R)+M\right]^{1 / m}
$$

В случае справедливости (1.24), (1.26) при произвольном $b>0$ и подходящем значении $M$

$$
\left[u_{b}^{m}(r)-M\right]_{+}^{1 / m} \leqslant u_{0}(r) .
$$

Наконец, пусть выполнено (1.27). Тогда при $\nu>0$ положим $\frac{1}{m} \theta_{N} c_{N}^{1-m} b^{\frac{m-p}{2} \lambda}=\nu$, откуда $b=\left[\nu m /\left(\theta_{N} c_{N}^{1-m}\right)\right]^{\frac{2}{(m-p) \lambda}}$. При достаточно малом значении $\varepsilon>0$ и подходящем выборе $M$

$$
\left[u_{b-\varepsilon}^{m}(r)-M\right]_{+}^{1 / m} \leqslant u_{0}(r) \leqslant\left[u_{b+\varepsilon}^{m}(r)+M\right]^{1 / m} .
$$

В случае $\nu=0$ вместо (4.10) имеют место неравенства

$$
\left[u^{m}(r ; \varepsilon)-M\right]_{+}^{1 / m} \leqslant u_{0}(r) \leqslant\left[u_{\varepsilon}^{m}(r)+M\right]^{1 / m} .
$$

При $\nu<0$ положим $-\frac{1}{m} q_{N} R^{\lambda} c_{N}^{1-m}=\nu$, откуда $R=\left\{|\nu| m /\left(c_{N}^{1-m} q_{N}\right)\right]^{1 / \lambda}$. Выбирая теперь $\varepsilon$ и $1 / M$ положительными и достаточно мальми, получаем соотношения

$$
\left[u^{m}(r ; R-\varepsilon)-M\right]_{+}^{1 / m} \leqslant u_{0}(r) \leqslant\left[u^{m}(r ; R+\varepsilon)+M\right]^{1 / m} .
$$

ДоКАЗАТЕЛЬСТво ТЕОРЕМЫ 6 . Пусть $x_{0}-$ произвольная точка шара $B_{d}$ и $\delta-$ произвольное положительное число, меньшее $R-d$. Тогда, очевидно, $B_{d+\delta} \subset B_{R}$. Так как $u(r, t)$ сходится к $u(r ; R)$ равномерно на множестве $B_{R}$, то существует такое значение $t_{0}>0$, что $u(x, t) \leqslant \varepsilon^{\frac{\gamma}{m}} c_{N} \delta^{\frac{2}{m-p}}$ для $(x, t) \in Q=B_{d+\delta} \times\left[t_{0}, \infty\right)$, где $\gamma=(m-p) /(1-p)$, а $\varepsilon$ - единственный положительный корень уравнения $s^{\gamma}=1-s$. Далее отдельно рассмотрим два случая $p<m<1$ и $m>1$. Пусть сначала $m>1$. Введем вспомогательную функцию

$$
w_{1}(x, t)=\varepsilon^{\frac{\gamma}{m}}\left\{\left[k_{0}-(1-p)\left(t-t_{0}\right)\right]_{+}^{\frac{m}{1-p}}+c_{N}^{m}\left|x-x_{0}\right|^{\frac{2 m}{m-p}}\right\}^{\frac{1}{m}} .
$$


Здесь $k_{0}=\left[c_{N} \delta^{\frac{2}{m-p}}\right]^{1-p}$. Несложно проверить, что $w_{1}(x, t) \geqslant u(x, t)$ на параболической границе $Q$ и для $w_{1}(x, t)$ выполнено неравенство (3.4). По теореме сравнения $u(x, t) \leqslant w_{1}(x, t)$ в $Q$. Следовательно, $u\left(x_{0}, t\right)=0$ при $t \geqslant t_{0}+k_{0} /(1-p)$ в любой точке $x_{0} \in B_{d}$. В случае $p<m<1$ доказательство проводится аналогичньм образом, только вместо $w_{1}(x, t)$ следует рассмотреть

$$
w_{2}(x, t)=\varepsilon\left\{\left[k_{0}-(1-p)\left(t-t_{0}\right)\right]_{+}^{\frac{1}{1-p}}+c_{N}\left|x-x_{0}\right|^{\frac{2}{m-p}}\right\} .
$$

Автор благодарен рецензенту за замечания, способствовавшие устранению неточностей и улучшению содержания работы.

\section{Список литературы}

1. Калашников $A$. C. Некоторые вопросы качественной теории нелинейных вырождающихся параболических уравнений второго порядка // УМН. 1987. Т. 42. № 1. С. 135-176.

2. Herrero M.A., Velazquez J. J.L. On the dynamics of a semilinear heat equation with strong absorption // Comm. Partial Differential Equations. 1989. V. 14. № 12 . P. $1653-1715$.

3. Калашников A. C. О квазилинейных вырождающихся параболических уравнениях с сингулярньми младшими членами и растущими начальными данными // Дифференц. уравнения. 1993. Т. 29. №6. С. 999-1009.

4. Herrero M. A., Pierre M. The Cauchy problem for $u_{t}=\Delta u^{m}$ when $0<m<1 / /$ Trans. Amer. Math. Soc. 1985. V. 291. № 1. P. 145-158.

5. Гладков $A$. Л. О неограниченных решениях нелинейного уравнения теплопроводности с сильной конвекцией на бесконечности // ЖВМ и МФ. 1996. Т. 36. № 10. С. 73-86.

6. Kamin S., Peletier L.A., Vazquez J. L. A nonlinear diffusion-absorption equation with unbounded initial data. Nonlinear diffusion equations and their equilibrium states. Proc. 3rd Conf., Gregynog/UK, 1989 // Progr. Nonlinear Differential Equations Appl. 1992. V. 7. P. 243-263.

7. McLeod J. B., Peletier L. A., Vazquez J. L. Solutions of a nonlinear ODE appearing in the theory of diffusion and absorption // Differential and Integral Equations. 1991. V. 4. № 1. P. 1-14.

8. Гладков А. Л. Задача Коши для некоторых вырождающихся квазилинейных параболических уравнений с поглощением // Сиб. матем. журн. 1993. Т. 34. № 1. С. 47-64.

9. Vazquez J.L., Walias M. Existence and uniqueness of solutions of diffusion-absorption equations with general data // Differential and Integral Equations. 1994. V. 7. № 1. P. $15-36$.

10. Gilding B. H., Kersner $R$. The characterization of reaction-convection-diffusion processes by travelling waves // J. Differential Equations. 1996. V. 124. № 1. P. 27-79.

11. Гладков А. Л. О задаче Коши в классах растущих функций для уравнения фильтрации с конвекцией // Матем. сб. 1995. Т. 186. №6. С. 35-56.

Витебский государственный университет,

Поступила в редакцию

г. Витебск, Беларусь

E-mail: gladkov@vgpi.belpak.vitebsk.by

26.01 .1998 и 18.03 .1999 